\title{
Spectrum of childhood interstitial and diffuse lung diseases at a tertiary hospital in Egypt
}

\author{
Salma G. Abdelhady $\mathbb{1}^{1,2}$, Eman M. Fouda ${ }^{1,2}$, Malak A. Shaheen ${ }^{1,2}$, \\ Faten A. Ghazal ${ }^{3,4}$, Ahmed M. Mostafa ${ }^{5,6}$, Ahmed M. Osman ${ }^{7}$, \\ Andrew G. Nicholson ${ }^{8,9}$ and Heba M. Hamza ${ }^{1,2}$
}

Affiliations: ${ }^{1}$ Dept of Paediatrics, Faculty of Medicine, Ain Shams University, Cairo, Egypt. ${ }^{2}$ Children's Hospital, Pulmonology Unit, Ain Shams University Hospitals, Cairo, Egypt. ${ }^{3}$ Dept of Pathology, Faculty of Medicine, Ain Shams University, Cairo, Egypt. "Pathology Lab, Ain Shams University Hospitals, Cairo, Egypt. ${ }^{5}$ Dept of Thoracic Surgery, Faculty of Medicine, Ain Shams University, Cairo, Egypt. ${ }^{6}$ Cardiovascular and Thoracic Academy, Ain Shams University Hospitals, Cairo, Egypt. ${ }^{7}$ Dept of Radiology, Faculty of Medicine, Ain Shams University, Cairo, Egypt. ${ }^{8}$ Dept of Histopathology, Royal Brompton and Harefield NHS Foundation Trust, London, UK. ${ }^{9}$ National Heart and Lung Institute, Imperial College, London, UK.

Correspondence: Salma Abdelhady, Children's Hospital, Ain Shams University, Abbassiah, Cairo, Egypt. E-mail: salmagamalhadyagmail.com

\section{ABSTRACT}

Background: Childhood interstitial and diffuse lung diseases (chILD) encompass a broad spectrum of rare pulmonary disorders. In most developing Middle Eastern countries, chILD is still underdiagnosed. Our objective was to describe and investigate patients diagnosed with chILD in a tertiary university hospital in Egypt. Methods: We analysed data of consecutive subjects (aged $<18$ years) referred for further evaluation at the Children's Hospital, Ain Shams University (Cairo, Egypt). Diagnosis of chILD was made in accordance with the ChILD-EU criteria. The following information was obtained: demographic data, clinical characteristics, chest computed tomography findings, laboratory studies, spirometry, bronchoalveolar lavage and histopathology findings.

Results: 22 subjects were enrolled over 24 months. Median age at diagnosis was 7 years (range 3.5-14 years). The most common manifestations were dyspnoea (100\%), cough (90.9\%), clubbing (95.5\%) and tachypnoea (90.9\%). Systematic evaluation led to the following diagnoses: hypersensitivity pneumonitis $(n=3)$, idiopathic interstitial pneumonias $(n=4)$, chILD related to chronic granulomatous disease $(n=3)$, chILD related to small airways disease $(n=3)$, post-infectious chILD $(n=2)$, Langerhans cell histiocytosis $(n=2)$, idiopathic pulmonary haemosiderosis $(n=2)$, granulomatous lymphocytic interstitial lung disease $(n=1)$, systemic sclerosis $(n=1)$ and familial interstitial lung disease $(n=1)$. Among the subjects who completed the diagnostic evaluation $(n=19)$, treatment was changed in $13(68.4 \%)$ subjects.

Conclusion: Systematic evaluation and multidisciplinary peer review of chILD patients at our tertiary hospital led to changes in management in $68 \%$ of the patients. This study highlights the need for an Egyptian chILD network with genetic testing, as well as the value of collaborating with international groups in improving healthcare for children with chILD.

@ERSpublications

In Egypt, childhood interstitial and diffuse lung diseases (chILD) are still underdiagnosed. Establishment of an Egyptian chILD network with genetic testing is essential to improve healthcare for children diagnosed with chILD. https://bit.ly/385qKsU

Cite this article as: Abdelhady SG, Fouda EM, Shaheen MA, et al. Spectrum of childhood interstitial and diffuse lung diseases at a tertiary hospital in Egypt. ERJ Open Res 2021; 7: 00880-2020 [https:// doi.org/10.1183/23120541.00880-2020].

This article has supplementary material available from openres.ersjournals.com

Received: 25 Nov 2020 | Accepted: 24 Feb 2021

Copyright $\odot$ The authors 2021. This version is distributed under the terms of the Creative Commons Attribution NonCommercial Licence 4.0. For commercial reproduction rights and permissions contact permissions@ersnet.org 


\section{Introduction}

Childhood interstitial and diffuse lung disease (chILD) is a term that describes a rare heterogeneous group of diffuse parenchymal lung diseases associated with considerable morbidity and mortality [1]. ChILD typically presents with tachypnoea, hypoxaemia, retractions, crackles and failure to thrive [2]. The causes of chILD are numerous; they include toxic exposures, immune deficiency, systemic diseases, infections and genetic causes. In several cases the exact aetiology remains unknown [3]. Diagnostic testing to determine the exact cause of chILD has many merits, such as avoiding unnecessary empirical treatment, initiating disease-specific treatment and guiding discussions with families regarding disease prognosis and duration of therapy. A systematic approach is recommended to identify the specific chILD diagnosis, starting with the clinical assessment, echocardiography and blood tests. Lung function testing may be helpful [2]. Chest computed tomography (CT) is considered the standard modality for radiological investigation, as it confirms the diagnosis of chILD, identifies the disease extent and may allow diagnosis without biopsy [4]. In addition, genetic testing is helpful in making the diagnosis and evaluating the recurrence risk for affected families [5]. If the diagnosis can't be made at this point, then invasive tests are required. Bronchoalveolar lavage (BAL) may be helpful in diagnosis of certain conditions such as pulmonary haemorrhage syndromes, Langerhans cell histiocytosis (LCH), pulmonary alveolar proteinosis, aspiration syndromes and, most importantly, exclusion of infections [6]. Histopathological assessment of lung biopsies is an important diagnostic tool when other investigations have not identified the precise chILD diagnosis [2]. At our hospital, we face many obstacles regarding the diagnosis of chILD cases; most importantly the lack of a structured multidisciplinary approach. Before this study, the diagnostic evaluation for chILD was not unified in all cases and lung biopsies were not performed due to lack of facilities and expertise to interpret the results. The purpose of this study was to reach the specific chILD entity by application of a systematic diagnostic evaluation for chILD cases referred at our hospital.

\section{Methods}

We performed an observational study of children diagnosed with chILD at the paediatric pulmonology section of the Children's Hospital, Ain Shams University (Cairo, Egypt) over a 2-year period (2018-2020). At the time of enrolment, all patients were subject to a standardised diagnostic evaluation, aiming to reach a specific chILD diagnosis. Baseline data were collected just prior to the diagnostic evaluation. On presentation to our hospital, fully informed parental consent was obtained prior to study inclusion. The study was approved by the ethics committee of Ain Shams University.

The inclusion criterion was clinical diagnosis of chILD syndrome, i.e. satisfying the 2015 European taskforce's definition of chILD [2]. All enrolled subjects had at least three of the following four criteria: 1) respiratory symptoms (cough, dyspnoea, exercise intolerance); 2) respiratory signs (resting tachypnoea, retractions, respiratory failure, clubbing, failure to thrive); 3) hypoxaemia (oxygen saturation <90\%); and 4) diffuse radiological abnormalities. Patients with common causes of diffuse lung disease (i.e. cystic fibrosis, primary ciliary dyskinesia and congenital heart disease) were excluded from the study.

Data collected at baseline included patient demographics, family and neonatal history, initial and current history and symptoms, as well as the physical examination findings. Guided by history and physical examination, the tests indicated were performed sequentially, starting with noninvasive tests, such as blood tests, echocardiography, chest CT scan and lung function tests. FAN et al.'s [7] severity score was recorded for each subject on presentation as follows: 1) asymptomatic; 2) symptomatic, normal oxygen saturation under all conditions; 3) symptomatic, normal resting room air saturation, but hypoxaemia $<90 \%$ with exercise or sleep; 4) symptomatic, hypoxaemia $<90 \%$ at rest; and 5) symptomatic, hypoxaemia at rest and pulmonary hypertension. Blood tests were guided by the clinical assessment and they included complete blood picture, selected immune studies, hypersensitivity pneumonitis (HP) precipitins panel and viral serology (for Epstein-Barr virus, cytomegalovirus and HIV). Genetic testing for chILD is not available in Egypt, and thus it was not performed as a part of this study. Plain chest radiography and CT scans were performed for all enrolled subjects (volumetric scan during inspiration, with a high-resolution fine-cut spaced expiratory scan). The images were interpreted by a consultant radiologist specialised in thoracic imaging (AMO). If a specific diagnosis could not be established at this point, we then proceeded to invasive testing. BAL was not performed as a routine procedure; it was indicated to investigate infectious agents or if diffuse alveolar haemorrhage was suspected, thereby potentially avoiding biopsy. Lung biopsy was performed when a specific diagnosis could not be achieved through the methods described earlier. The lung biopsy procedure was performed according to the chILD European protocols [2], via the open surgical technique. The obtained tissue was divided as follows: $20 \%$ was sent as a fresh tissue for microbial cultures (bacterial, mycobacterial, viral and fungal) and $80 \%$ was fixed using formalin to form wax blocks. Haematoxylin and eosin staining was routinely performed for all lung biopsy specimens, and further special stains were ordered as required. Electron microscopy studies for lung biopsy specimens were not done, as they are not available in our hospital. As no chILD clinical or research network previously existed 
TABLE 1 Classification of enrolled subjects according to their clinical, radiological, laboratory and histopathology features

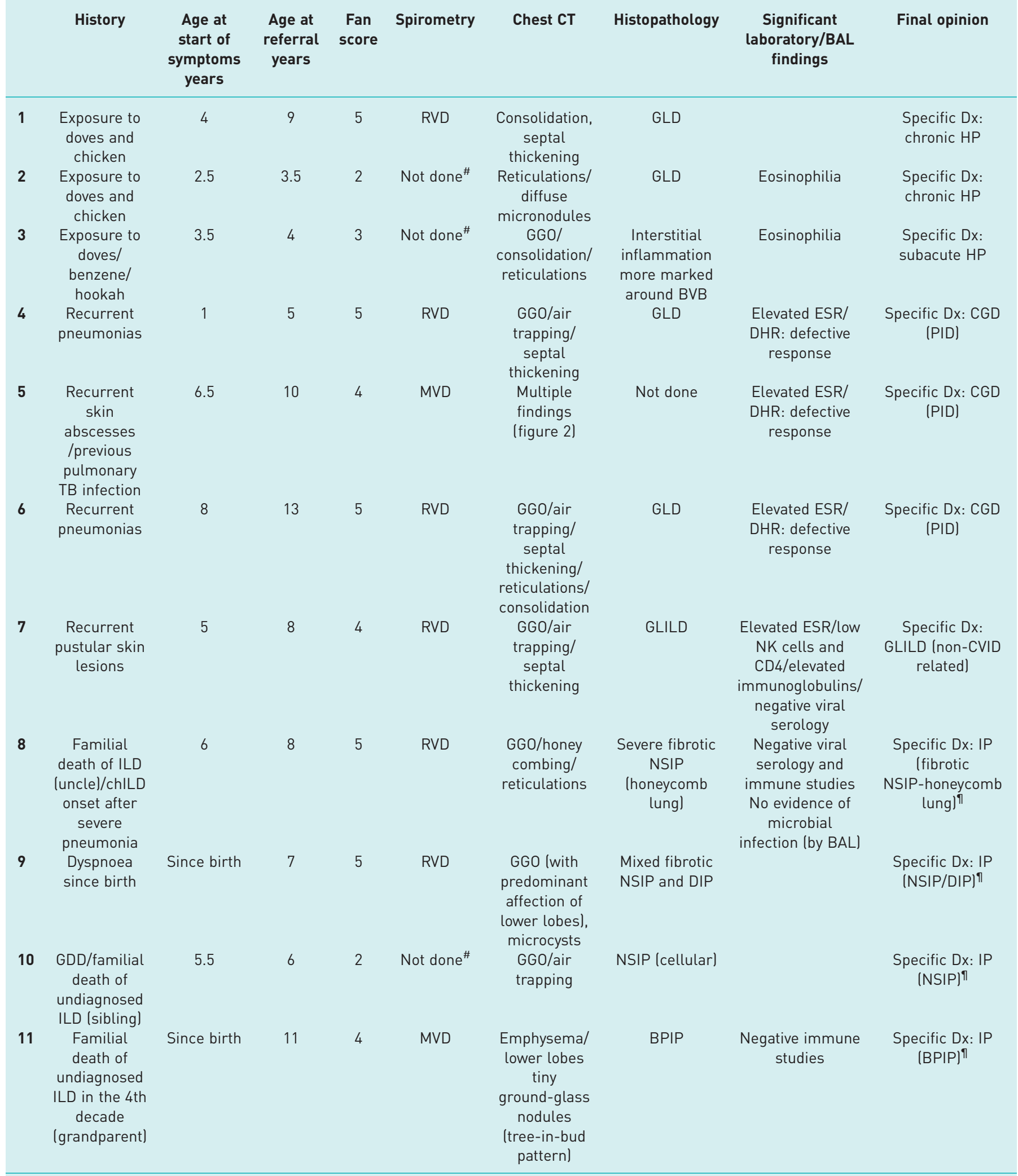


TABLE 1 Continued

\begin{tabular}{|c|c|c|c|c|c|c|c|c|c|}
\hline & History & $\begin{array}{l}\text { Age at } \\
\text { start of } \\
\text { symptoms } \\
\text { years }\end{array}$ & $\begin{array}{l}\text { Age at } \\
\text { referral } \\
\text { years }\end{array}$ & $\begin{array}{l}\text { Fan } \\
\text { score }\end{array}$ & Spirometry & Chest CT & Histopathology & $\begin{array}{c}\text { Significant } \\
\text { laboratory/BAL } \\
\text { findings }\end{array}$ & Final opinion \\
\hline 12 & $\begin{array}{c}\text { Familial } \\
\text { death of } \\
\text { undiagnosed } \\
\text { ILD in the 4th } \\
\text { decade } \\
\text { (grandparent) }\end{array}$ & Since birth & 5 & 5 & RVD & $\begin{array}{l}\text { Air trapping/ } \\
\text { hyperinflation/ } \\
\text { lower lobes } \\
\text { tiny } \\
\text { ground-glass } \\
\text { nodules } \\
\text { (tree-in-bud } \\
\text { pattern) }\end{array}$ & $\begin{array}{l}\text { Parents } \\
\text { refused (cases } \\
11 \text { and } 12 \text { are } \\
\text { siblings) }\end{array}$ & $\begin{array}{c}\text { Negative immune } \\
\text { studies }\end{array}$ & $\begin{array}{l}\text { Suggestive Dx: } \\
\text { familial ILD of } \\
\text { unidentified } \\
\text { aetiology }\end{array}$ \\
\hline 13 & GDD & Since birth & 8 & 4 & Not done & $\begin{array}{l}\text { GGO/air } \\
\text { trapping }\end{array}$ & $\begin{array}{c}\text { Chronic } \\
\text { bronchiolitis/ } \\
\text { interstitial } \\
\text { chronic } \\
\text { inflammation }\end{array}$ & & $\begin{array}{l}\text { Specific Dx: } \\
\text { chILD related to } \\
\text { SAD with } \\
\text { background } \text { IP }^{+}\end{array}$ \\
\hline 15 & $\begin{array}{c}\text { NICU } \\
\text { admission at } \\
\text { birth for } \\
2 \text { months/full } \\
\text { term }\end{array}$ & Since birth & 14 & 2 & MVD & $\begin{array}{l}\text { GGO la few } \\
\text { show crazy } \\
\text { pavingl }\end{array}$ & $\begin{array}{c}\text { Chronic } \\
\text { bronchiolitis/ } \\
\text { interstitial } \\
\text { chronic } \\
\text { inflammation }\end{array}$ & & $\begin{array}{l}\text { Specific Dx: } \\
\text { chILD related to } \\
\text { SAD with } \\
\text { background } \mathrm{IP}^{+}\end{array}$ \\
\hline 16 & $\begin{array}{l}\text { Puffy fingers/ } \\
\text { digital tip } \\
\text { ulcers/ } \\
\text { sclerodactyly/ } \\
\text { induration } \\
\text { proximal to } \\
\text { MCP (late) }\end{array}$ & 4 & 8 & 4 & Not done ${ }^{\#}$ & Bibasilar GGO & $\begin{array}{c}\text { BPIP + focal } \\
\text { OP }\end{array}$ & $\begin{array}{l}\text { Abnormal } \\
\text { nail-fold } \\
\text { capillaries }\end{array}$ & $\begin{array}{l}\text { Specific Dx: } \\
\text { Systemic } \\
\text { sclerosis }\end{array}$ \\
\hline 18 & $\begin{array}{l}\text { Haemoptysis/ } \\
\text { admitted six } \\
\text { times for } \\
\text { blood } \\
\text { transfusion } \\
\text { for severe } \\
\text { microcytic } \\
\text { anaemia }\end{array}$ & 5.5 & 7 & 2 & RVD & GGO & Not done & $\begin{array}{l}\text { BAL: HLM }>45 \% \\
\text { of cells/other } \\
\text { causes of DAH } \\
\text { were excluded }\end{array}$ & Specific Dx: IPH \\
\hline 19 & $\begin{array}{l}\text { Progressive } \\
\text { dyspnoea } \\
\text { over } \\
2 \text { months }\end{array}$ & 11 & 11 & 5 & Not done $e^{\#}$ & $\begin{array}{c}\text { Cysts (sparing } \\
\text { CPA)/GGO/tiny } \\
\text { nodules }\end{array}$ & $\begin{array}{l}\text { Death before } \\
\text { any invasive } \\
\text { tests }\end{array}$ & & $\begin{array}{l}\text { Suggestive Dx: } \\
\text { LCH }\end{array}$ \\
\hline 20 & & 1 & 3.5 & 4 & Not done $e^{\#}$ & $\begin{array}{c}\text { Cysts (sparing } \\
\text { CPA)/GGO }\end{array}$ & Dropped out & & $\begin{array}{c}\text { Suggestive Dx: } \\
\text { LCH }\end{array}$ \\
\hline
\end{tabular}




\section{TABLE 1 Continued}

\begin{tabular}{|c|c|c|c|c|c|c|c|c|c|}
\hline & History & $\begin{array}{l}\text { Age at } \\
\text { start of } \\
\text { symptoms } \\
\text { years }\end{array}$ & $\begin{array}{l}\text { Age at } \\
\text { referral } \\
\text { years }\end{array}$ & $\begin{array}{l}\text { Fan } \\
\text { score }\end{array}$ & Spirometry & Chest CT & Histopathology & $\begin{array}{c}\text { Significant } \\
\text { laboratory/BAL } \\
\text { findings }\end{array}$ & Final opinion \\
\hline 21 & $\begin{array}{l}\text { History of } \\
\text { pulmonary } \\
\text { TB }\end{array}$ & 3.5 & 4.5 & 5 & Not done ${ }^{\#}$ & $\begin{array}{l}\text { GGO/ } \\
\text { reticulations/ } \\
\text { atelectatic } \\
\text { bands }\end{array}$ & $\begin{array}{l}\text { Clinically } \\
\text { improving (not } \\
\text { done) }\end{array}$ & $\begin{array}{c}\text { Negative immune } \\
\text { studies }\end{array}$ & $\begin{array}{l}\text { Specific Dx: } \\
\text { post-infectious } \\
\text { chILD (post-TB) }\end{array}$ \\
\hline
\end{tabular}

CT: computed tomography; BAL: bronchoalveolar lavage; RVD: restrictive ventilatory dysfunction; GLD: granulomatous lung disease; Dx: diagnosis; HP: hypersensitivity pneumonitis; GGO: ground-glass opacities; BVB: bronchovascular bundles; ESR: erythrocyte sedimentation rate; DHR: dihydrorhodamine test; CGD: chronic granulomatous disease; PID: primary immune deficiency; TB: tuberculosis; MVD: mixed ventilatory dysfunction; GLILD: granulomatous lymphocytic interstitial lung disease; NK: natural killer; CVID: common variable immune deficiency; ILD: interstitial lung disease; chILD: childhood interstitial and diffuse lung diseases; NSIP: nonspecific interstitial pneumonia; IP: interstitial pneumonia; DIP: desquamative interstitial pneumonia; GDD: global developmental delay; BPIP: bronchiolocentric pattern of interstitial pneumonias; SAD: small airways disease; NICU: neonatal intensive care unit; MCP: metacarpophalangeal joint; OP: organising pneumonia; HLM: haemosiderin-laden macrophages; DAH: diffuse alveolar haemorrhage; IPH: idiopathic pulmonary haemosiderosis; CPA: costophrenic angle; LCH: Langerhans cell histiocytosis. \#: too young or too dyspnoeic to do the test or suffering from global delay

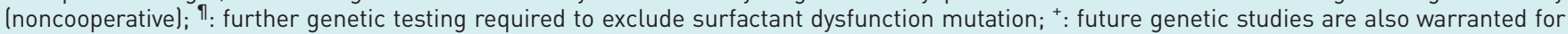
this group, as symptom onset was at birth or shortly after birth.

in Egypt, we chose to refer histological specimens for a second opinion overseas, which is a common practice among global chILD networks. Lung biopsy samples were reviewed independently by two consultant thoracic pathologists (FAG and AGN) and classified according to the global chILD schemes for paediatric interstitial and diffuse lung disease $[1,2,6,8,9]$. Cases with multiple or overlapping patterns were classified according to the dominant one, and any minor patterns were recorded. Further specific investigations were ordered for selected subjects guided by the histopathology results. Each individual case was subject to a multidisciplinary peer review to standardise diagnostic precision. Treatment plans were adjusted according to the corresponding final chILD diagnosis.

\section{Statistical analysis}

Quantitative nonparametric data are presented as median (interquartile range (IQR)). Qualitative variables are presented as $\mathrm{n}(\%)$. Height, weight and body mass index (BMI) were converted to Centers for Disease Control and Prevention z-scores and percentiles.

\section{Results}

Over the study period, 22 patients were identified. The median (IQR) age of symptom onset was 3.75 $(0-11)$ years, while the median (IQR) age at referral for diagnostic evaluation at our hospital was 7 (3.5-14) years. In eight (36.4\%) subjects, symptoms had started during the first 2 years of life, but specialist referral was delayed.

Dyspnoea (100\%, 22 out of 22$)$, cough $(90.9 \%, 20$ out of 22$)$ and recurrent pneumonias $(77.3 \%, 17$ out of 22) were the most frequent symptoms in all studied patients. All enrolled subjects reported dyspnoea on exertion, while 20 (90.9\%) subjects reported dyspnoea at rest as well. Cough was also a troublesome symptom. All 20 subjects who gave history of cough reported that their cough was dry; however, eight of them reported frequent episodes of productive cough as well. In addition, 10 (45.5\%) subjects had history of recurrent febrile episodes, refractory to routine antibiotic therapy. Furthermore, seven (31.8\%) patients were falsely diagnosed with childhood asthma by primary care physicians and they were referred later on due to poor response to treatment. Other symptoms included anorexia, attacks of cyanosis, weight loss, haemoptysis and systemic manifestations. Digital clubbing (95.5\%, 21 out of 22), tachypnoea (90.9\%, 20 out of 22), tachycardia $(68.2 \%, 15$ out of 22$)$ and failure to thrive $(54.5 \%, 12$ out of 22$)$ were the most commonly observed signs. Abnormal auscultatory findings included inspiratory fine crackles (90.9\%, 20 out of 22), wheeze $(68.2 \%, 15$ out of 22$)$ and loud S2 (36.4\%, eight out of 22). In addition, chest 
deformities were noticed in three (13.6\%) subjects. Median weight $\mathrm{z}$-score, height $\mathrm{z}$-score and BMI $\mathrm{z}$-score among studied subjects were low $(-2.17,-1.42$ and -1.36 , respectively). Furthermore, lower weight and height $\mathrm{z}$-scores were significantly associated with lower oxygen saturation measured by pulse oximetry levels in room air at rest and during sleep. Five (22.7\%) subjects had family history of interstitial lung disease (ILD). The clinical severity of the disease was assessed using the Fan scoring system and we found that five $(22.7 \%)$ of the studied patients were categorised as score 2; one (4.5\%) subject was categorised as score 3; eight (36.4\%) as score 4; and eight (36.4\%) as score 5. Among the study group, eight (36.4\%) subjects had pulmonary hypertension by echocardiography on initial assessment.

Plain chest radiography findings were generally nonspecific. A comparison between chest radiographs and corresponding chest CT findings among enrolled subjects is provided in supplementary table S1. The predominant abnormality identified by chest CT was the presence of ground-glass opacity (90.9\%). Other common abnormalities included septal thickening (50\%), air trapping (40.9\%), mosaic attenuation (31.8\%), reticulations $(27.3 \%)$ and consolidation $(22.7 \%)$. Less common features included tree-in-bud pattern, honeycomb cysts, nodules, air-filled cysts, hyperinflation, traction bronchiectasis, crazy paving, lymphadenopathy and emphysematous changes.

Only 14 (63.6\%) subjects were able to perform spirometry and the rest were too dyspnoeic or too young to perform the test. Of the subjects who could perform the test, nine $(64.3 \%)$ showed a restrictive pattern, three $(21.4 \%)$ showed a mixed obstructive and restrictive pattern and two (14.3\%) had normal spirometry results.

We managed to make a specific diagnosis without a lung biopsy in four patients. One patient was diagnosed with post-tuberculous chILD based on clinical evaluation and noninvasive tests, and another patient was diagnosed with chronic granulomatous disease (CGD) based on the result of dihydrorhodamine (DHR) testing. Two other patients had a history of recurrent hospitalisation for severe microcytic hypochromic anaemia requiring blood transfusion, following several attacks of haemoptysis,
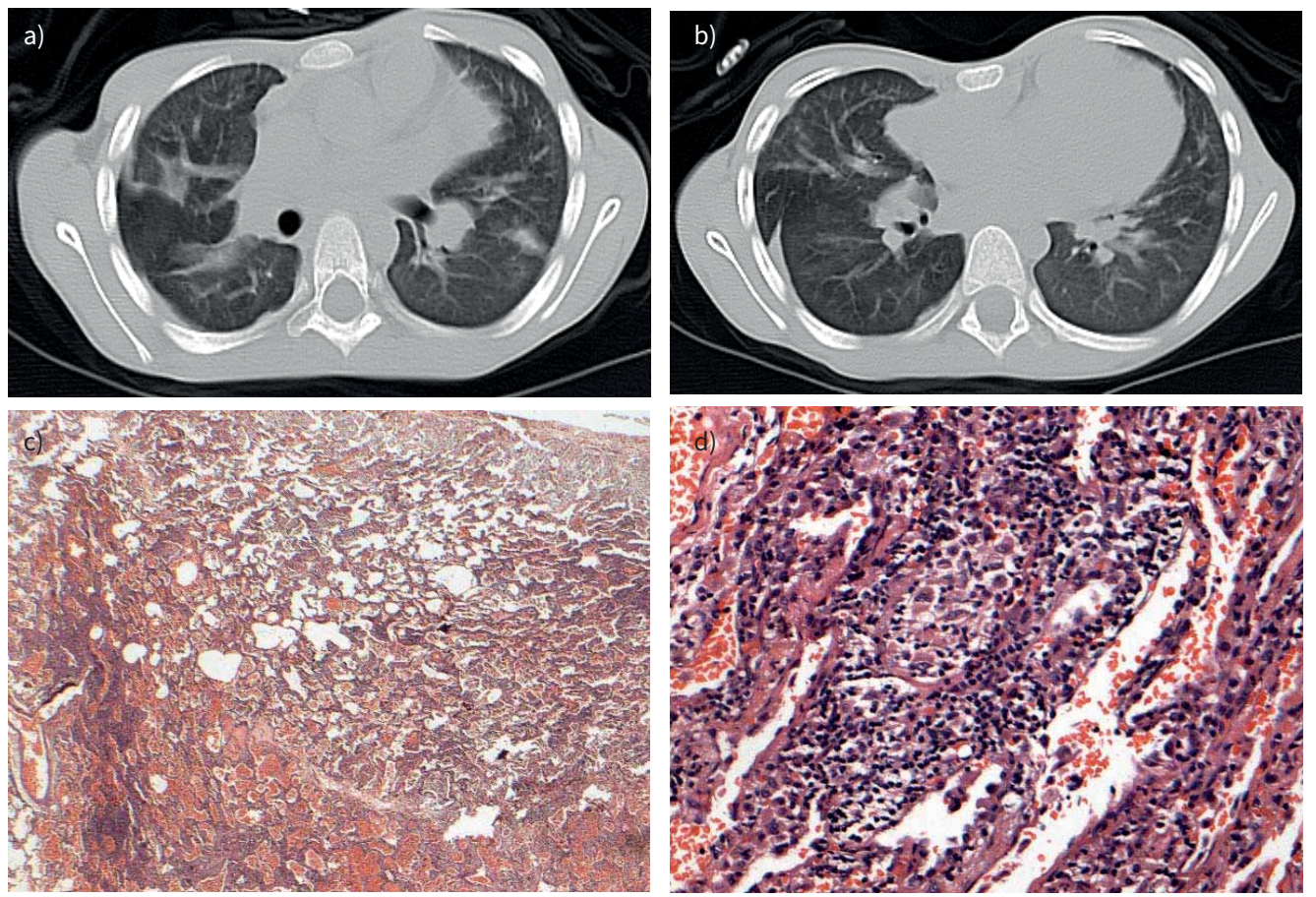

FIGURE 1 Subject 1: 9-year-old male referred with possible diagnosis of childhood interstitial and diffuse lung disease. He complained of recurrent attacks of dyspnoea and dry cough. He had a strong history of exposure to birds. On presentation, he was tachypnoeic and hypoxic loxygen saturation $88 \%$ at rest in room air). He had marked failure to thrive with first-degree clubbing, and auscultation revealed widespread fine crepitations. Echocardiography showed dilated right side with pulmonary hypertension. a) Axial computed tomography shows bilateral scattered subsegmental consolidative patches associated with few scattered atelectatic bands; b) interlobular and fissural thickening; c) bronchocentric chronic inflammation (low power); d) small poorly formed granuloma (high power) (haematoxylin and eosin $c$ ) $\times 20, d$ ) $\times 200$ ). Overall, the features are consistent with hypersensitivity pneumonitis. There was a significant clinical improvement on elimination of bird exposure and systemic steroids. 

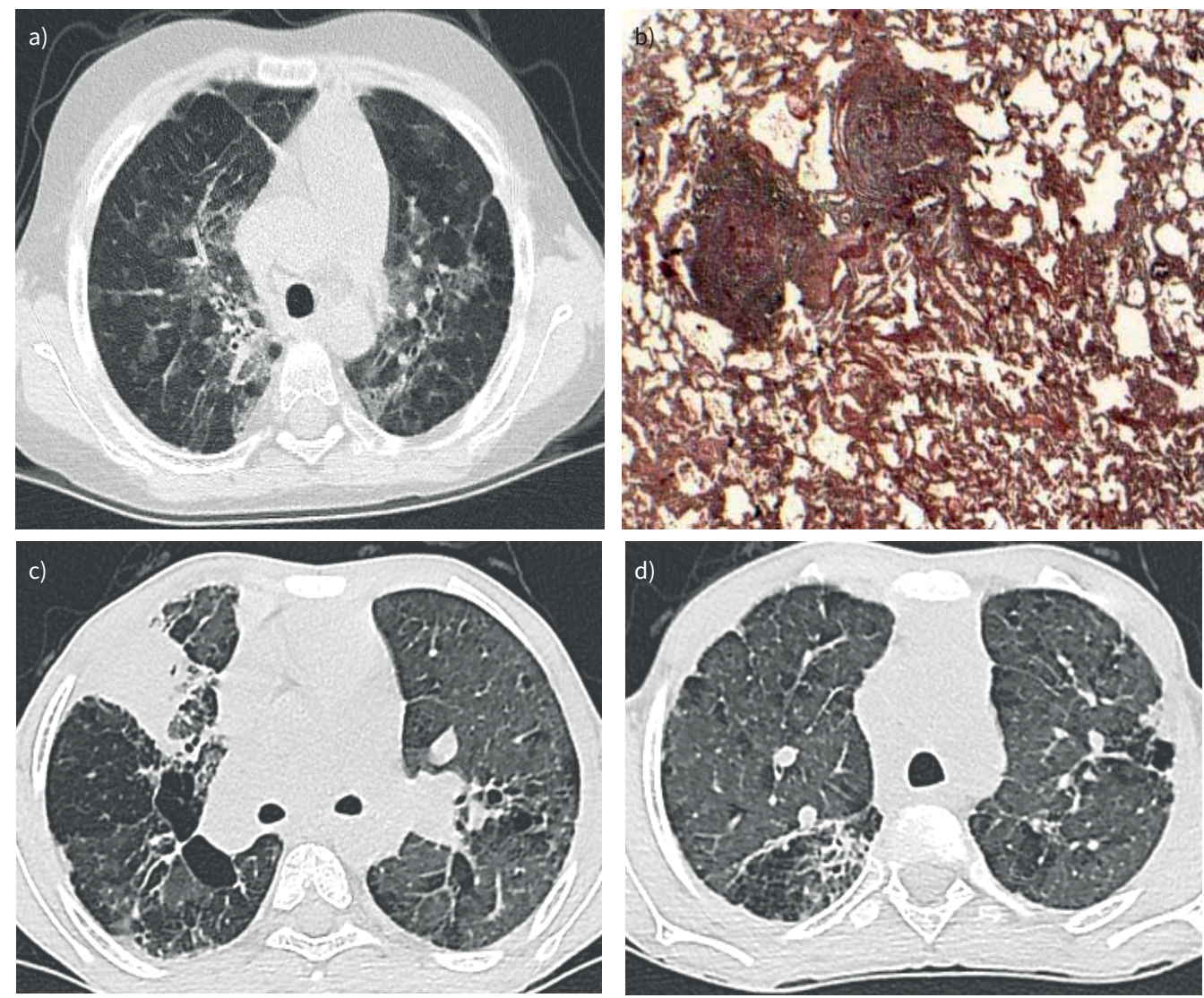

FIGURE 2 a, b) Subject 4: 5-year-old female patient, presented with severe failure to thrive and third-degree clubbing. She had profound hypoxaemia loxygen saturation $80 \%$ at rest in room air), with widespread fine crepitations and severe pulmonary hypertension. a) Axial computed tomography (CT) shows bilateral scattered central and peripheral patchy areas of ground-glass opacities (GGO) and septal thickening. This is associated with a few areas of air trapping, giving a mosaic pattern; b) lung histopathology shows granulomas surrounded by a rim of chronic inflammatory cells (haematoxylin and eosin $\times 20$ ). Thorough immunological testing was performed after the biopsy, and dihydrorhodamine (DHR) testing showed an abnormal response, confirming the diagnosis of chronic granulomatous disease. c, d) Subject 5: a 10-year-old male patient who presented with childhood interstitial and diffuse lung disease and was later diagnosed with chronic granulomatous disease (CGD). He had severe initial clinical presentation with hypoxia at rest (in room air) and marked failure to thrive. c) Axial CT shows bilateral peripheral and central large patchy areas of GGO with areas of air trapping giving a mosiac pattern, associated with septal thickening and a few mainly lower lobar emphysematous changes and central cystic changes. A large consolidative patch is noted affecting the anterior segment of the right upper lobe; d) abnormal bronchial thickening and minimal tractional bronchiectasis. Biopsy was planned, but it was deferred as he started to suffer from recurrent skin and deep-organ abscesses requiring surgical drainage. Further immune tests were done and DHR testing showed an abnormal response, confirming the diagnosis of CGD.

and they were diagnosed with idiopathic pulmonary haemosiderosis (IPH) by BAL after exclusion of all other causes of diffuse alveolar haemorrhage. More details regarding the BAL findings are provided in supplementary table S2.

Open lung biopsy was performed in 15 (68.2\%) subjects. Median (IQR) duration of post-operative intensive care unit admission was $1(1-4)$ days and median (IQR) post-operative duration of hospital stay was 6 (3-14) days. To treat any potential post-operative air-leak, a routine intercostal drainage was left in situ in all subjects. It was removed 1-2 days post-operation if there was no evidence of ongoing air-leak. Five $(33.3 \%)$ children experienced complications following the procedure. Three (20\%) had pneumothorax with underlying lung collapse requiring ongoing chest drainage for a median (IQR) 8 (7-21) days. One (6.7\%) of these children subsequently developed pneumonia requiring parenteral antibiotic therapy and one subject had persistent pneumothorax and was discharged 7 days post-operation on Heimlich valve for another 2 weeks. Two (13.3\%) subjects developed acute exacerbation shortly after the biopsy procedure, requiring systemic steroid therapy. No mortalities, haemorrhage, wound infection or need for intubation were recorded following the procedure.

The diagnostic evaluation was incomplete in three subjects. Two patients had imaging suggestive of LCH, but were lost after enrolment into the study (one death and one dropout). The third patient refused to have the lung biopsy; however, a suggestive diagnosis of familial ILD of unidentified aetiology was made based on history, examination and noninvasive tests. 



FIGURE 3 Subject 7: 8-year-old female had history of persistent dry cough and dyspnoea. She is the second child of first-degree consanguineous parents. In addition to the pulmonary symptoms, she had bouts of self-limiting noninfective diarrhoea and a) pustular skin lesions. In addition, she had a history of paediatric intensive care unit admission with severe sepsis which marked the disease onset. On presentation to us, she was tachypnoeic and hypoxic with widespread fine crepitations and wheeze by auscultation. An immunological profile showed elevated immunoglobulins and transient positivity of perinuclear anti-neutrophil cytoplasmic antibodies, which disappeared later on. Cluster of differentiation markers show borderline low natural killer cells and CD4 levels. Flow spirometry showed a restrictive pattern. b) Axial computed tomography shows bilateral mosaic appearance of both lung lobes (ground-glass appearance alternating with areas of air trapping), with peri-bronchial wall thickening and pre-septal thickening. c) Histopathology shows bronchocentric inflammation and granulomatous inflammation. d) Granuloma (high power). Haematoxylin and eosin c) $\times 40$ and d) $\times 200$. Histopathology findings were consistent with a granulomatous lymphocytic interstitial lung disease spectrum. Dihydrorhodamine test was normal and repeat immune studies showed persistent elevation of immunoglobulin levels, excluding underlying common variable immune deficiency.

Enrolled subjects were classified according to their clinical, radiological, laboratory and histopathology features (table 1; figures 1-8).

Among the subjects who completed the diagnostic evaluation $(\mathrm{n}=19)$, treatment was changed in 13 (68.4\%) subjects. Although the mainstay of treatment before and after the diagnostic evaluation remained systemic corticosteroids, it added value as we were able to plan the duration of steroid therapy, and changed our perspective in management of subsequent exacerbations. Highlights of changes to treatment lines after the diagnostic evaluation are shown in supplementary table S3.

\section{Discussion}

chILD is a rare disease, with few cases reported in each specialised centre annually. This is the first study to describe the diagnoses and characteristics of patients with chILD from one of the largest university hospitals in Egypt. Our study shows that systematic multidisciplinary review led to changes in management in the majority of cases diagnosed with chILD.

22 patients were referred to us from other hospitals for further evaluation, over a 24-month period. Although all enrolled subjects were aged $>2$ years at the time of referral to our hospital, the symptoms started during the first 2 years of life in one-third of them. However, referral to a tertiary hospital was delayed, reflecting inadequate awareness of chILD among general paediatricians. Age of symptom onset is particularly important, as it may provide a clue to the specific chILD subtype. chILD in infants aged $<2$ years is more commonly related to developmental disorders, pulmonary interstitial glycogenosis and neuroendocrinal hyperplasia of infancy. chILD presenting after 2 years is more likely to be related to 




FIGURE 4 Subject 8: 8-year-old female patient referred for further assessment. She complained of chronic dry cough and progressive dyspnoea. She has a history of familial death with undiagnosed interstitial lung disease in the fifth decade (uncle). On presentation to us she was tachycardic, tachypnoeic and hypoxic loxygen saturation $88 \%$ in room air). In addition, she had severe cachexia with third-degree clubbing. Echocardiography showed severe pulmonary hypertension with dilated right atrium and ventricle. a, b) Axial computed tomography shows bilateral predominantly upper lobar and peripheral ground-glass opacities associated with peripheral reticular infiltration and honeycombing. c) Histopathology shows that the lung architecture is completely lost, with replacement by end-stage (honeycomb) fibrosis (haematoxylin and eosin $\times 40$ ).

environmental exposures, systemic diseases and immune deficiency. Surfactant protein dysfunction mutations could present during infancy or later during childhood [10].

The clinical presentation of chILD is often nonspecific and overlaps with many conditions. Among our study group the most common symptoms were dyspnoea, cough and recurrent pneumonias. Although cough reported among chILD patients is classically dry [11], many of our cases reported superimposed attacks of productive cough, which may have accompanied intercurrent infective exacerbations or development of traction bronchiectasis. In addition, almost one-third of our patients were initially diagnosed with childhood asthma at primary care, and this should remind paediatricians to assess carefully difficult-to-treat asthma cases for the possibility of an alternative diagnosis. In addition, recurrent unexplained febrile episodes were common among our study group; this finding was observed among other chILD cohorts as well [10], and it should alert pediatricians to the possibility of chILD. Our findings of clinical presentation were similar to those reported in other series [12-14].

We found that thorough history and physical examination provided important information that aided in interpreting radiology and histopathology results. For instance, granulomatous lung disease was found in five patients; two patients had history of recurrent pneumonias, and further immune studies confirmed the diagnosis of chronic granulomatous disease; while the other three patients had strong histories of exposure to birds with improvement on steroid therapy and exposure elimination, confirming the diagnosis of hypersensitivity pneumonitis. Thus, confirmation of the diagnosis requires a multidisciplinary teamwork between clinicians, radiologists and pathologists.

In our study, we found that chest CT was useful in confirming the diagnosis of chILD, as well as identifying the disease distribution and helped in identifying a suitable biopsy site. However, the imaging was suggestive of the diagnosis in only two cases and no specific diagnosis was reached depending on the radiology alone. The predominant abnormality identified by chest CT among our study group was the presence of ground-glass opacities. Similar findings are reported in the literature [10, 13].

Bronchoscopy and BAL have an increasing role in the diagnosis of chILD. The primary value of bronchoalveolar lavage fluid (BALF) analysis is exclusion of infection as the underlying cause of chILD. 

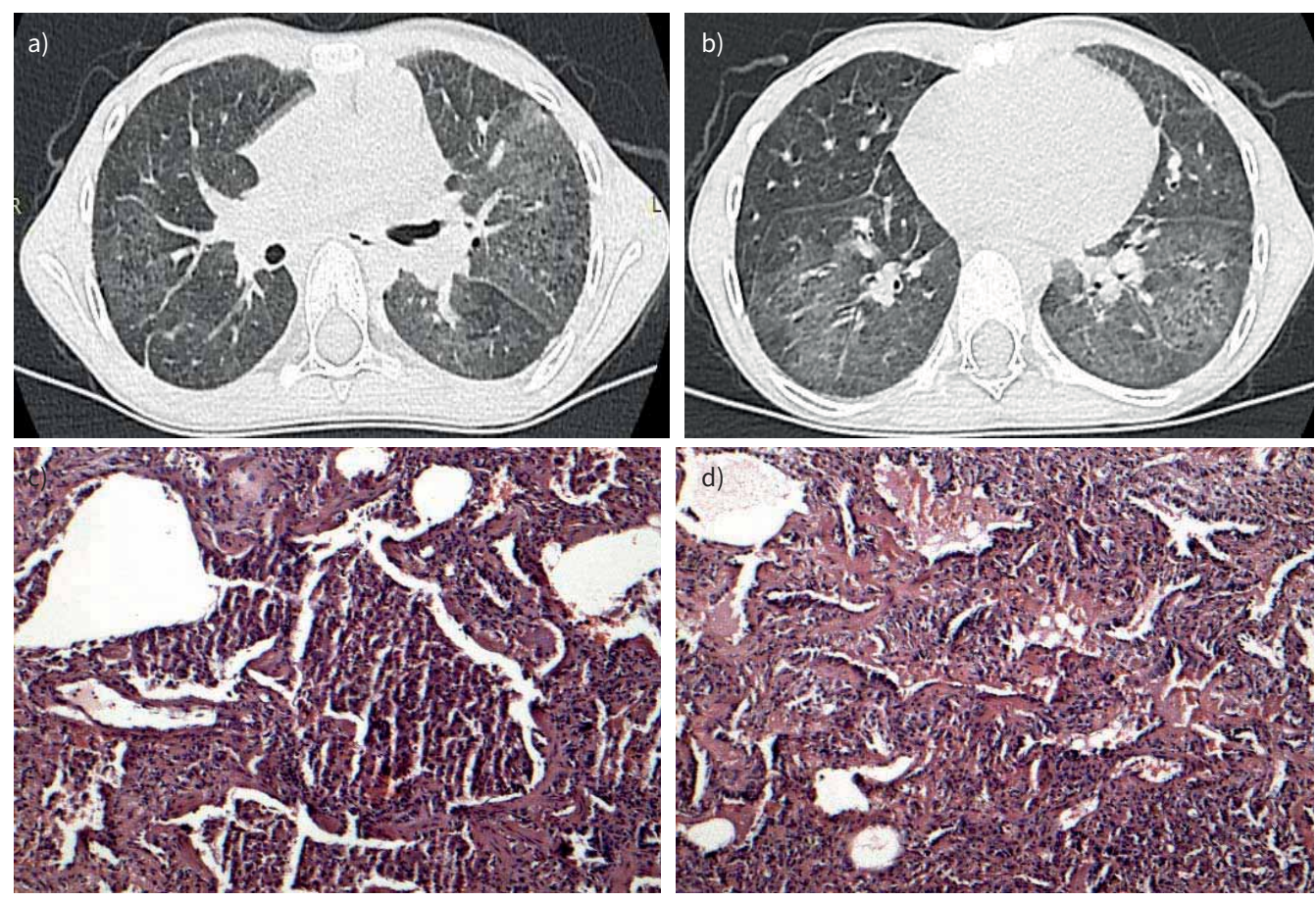

FIGURE 5 Subject 9: 7-year-old female, referred for further assessment. She had a history of dyspnoea shortly after birth, associated with failure to thrive. Her condition deteriorated to dyspnoea at rest and attacks of cyanosis. On presentation, she was markedly distressed, with respiratory rate 70 breaths $\mathrm{min}^{-1}$, heart rate 150 beats $\mathrm{min}^{-1}$ (tachycardia with gallop) and hypoxia loxygen saturation $85 \%$ in room air, at rest). In addition, she had third-degree clubbing and marked failure to thrive. She had bilateral fine crepitations on auscultation with accentuated second heart sound and tender hepatomegaly. Echocardiography showed dilated right ventricle and severe pulmonary hypertension. Axial computed tomography shows a) bilateral (predominantly upper lobar) subpleural and intraparenchymal microcysts and b) bilateral diffuse pulmonary ground-glass opacities, with predominant affection of both lower lobes. Histopathology shows c) predominance of alveolar macrophage accumulation and d) diffuse and uniform interstitial chronic inflammation and mild fibrosis, characteristic of nonspecific interstitial pneumonia. This mixed histological pattern (fibrotic nonspecific interstitial pneumonia and desquamative interstitial pneumonia) in the paediatric age group is suggestive of an underlying surfactant protein disorder (haematoxylin and eosin c, d) $\times 100$ ).

In addition, a high percentage of haemosiderin-laden macrophages in BALF is suggestive of diffuse alveolar haemorrhage syndromes, while BALF periodic acid-Schiff-positive granular material with hypocellularity is suggestive of alveolar proteinosis. Furthermore, positive CD1a staining of BALF cells is highly suggestive of pulmonary histiocytosis. Many other conditions may also be diagnosed using BAL, such as eosinophilic lung disease, aspiration syndromes and sarcoidosis [6]. Haemosiderin-laden macrophages were found in BALF of the two subjects diagnosed with IPH, and they constituted $>45 \%$ total cell count. Although the gold standard for IPH diagnosis is via lung biopsy, it is rarely performed as a BAL high percentage of haemosiderin-laden macrophages is considered a specific, sensitive and less invasive method for diagnosis of IPH in children [15].

Surgical lung biopsy plays an important role when other modalities fail to identify the specific chILD disease. Both open-lung biopsy (OLB) and video-assisted thoracoscopic surgery (VATS) have been used to obtain samples for histopathology. The American Thoracic Society Committee on chILD recommends VATS biopsy due to faster recovery time compared to OLB; however, the European guidelines do not make recommendations on the type of surgical approach $[2,6]$. In patients who had a diagnostic lung biopsy among our study group, we preferred the open surgical technique to ensure adequacy of the sampled tissue, and to avoid superficial nondiagnostic biopsies.

Among the subjects who had an OLB in our study group, a routine intercostal drainage was left in situ in all subjects to treat any potential post-operative air-leak. This practice is recommended as it was noticed that many children with chronic ILD develop a post-operative air-leak requiring chest drainage [16]. We report a shorter length of hospital stay than other comparable series in the literature, but the complication rate is fairly similar $[16,17]$. In our study, we found that the procedure of OLB was safe with no mortalities or significant adverse events, even in patients with hypoxaemia. The safety of the OLB procedure was similar to other published cohorts $[17,18]$. 



FIGURE 6 Subjects 11 and 12: two affected siblings with a history of grandparent death of undiagnosed interstitial lung disease (ILD) in the fourth decade. a, b) Computed tomography (CT) images from the older sibling: a) diffuse pan-lobar pulmonary emphysema with areas of air trapping, abnormal bronchial wall thickening; b) bilateral lower lobar scattered tiny ground-glass nodules showing tree-in-bud appearance. c, d) CT images from the younger sibling: c) air trapping and hyperinflation; d) bilateral few peripheral lower lobar tiny ground-glass nodules with a few of them giving tree-in-bud appearance. The younger sibling presented with a worse clinical picture (severity score 5) and her spirometry showed a restrictive defect. The older sibling presented with significant symptoms and signs and hypoxia at rest and her spirometry showed mixed obstructive and restrictive defect, with negative reversibility test. e, f) Histopathology of the older sibling, with findings consistent with idiopathic bronchiolocentric pattern of interstitial pneumonias: e) peribronchiolar inflammation (low power); f) peribronchiolar metaplasia (higher power) (haematoxylin and eosin e) $\times 40$ f) $\times 100$ ). Grocott stain for fungi was negative. General causes of diffuse parenchymal lung disease were thoroughly excluded as well as immune deficiency and $\alpha_{1}$-antitrypsin deficiency. A specific cause for this histological pattern couldn't be precisely identified, but a strong family history with two siblings having respiratory symptoms since birth and grandparent death due to ILD raises serious concerns for an underlying genetic disorder.

We report three confirmed cases of HP due to domestic bird exposure, which shows that environmental factors play an important role in the development of chILD in our study group. Although HP is still considered a rare disease in children, it has been increasingly reported in many chILD cohorts [12-19]. HP can be associated with exposure to a variety of finely dispersed environmental antigens, but avian exposure is by far the most common cause of HP in children, accounting for nearly two-thirds of cases [20].

To date, there is no consensus for the diagnosis of HP in children; however, HP is suggested if there is a history of exposure to an offending antigen, prominent radiological features and a positive precipitins test [19]. All three HP cases in our study had a strong history of exposure to birds, yet they tested negative for the HP precipitins testing panel. This finding might be attributed to the fact that standard HP panels are often irrelevant to the patient's environment [21]. Thus, patient-centred testing is recommended to avoid false negative results. The decision to refer for BAL was deferred, to avoid multiple invasive procedures requiring general anaesthesia, as BALF CD4/CD8 ratio can be within the normal range in children [22]. 

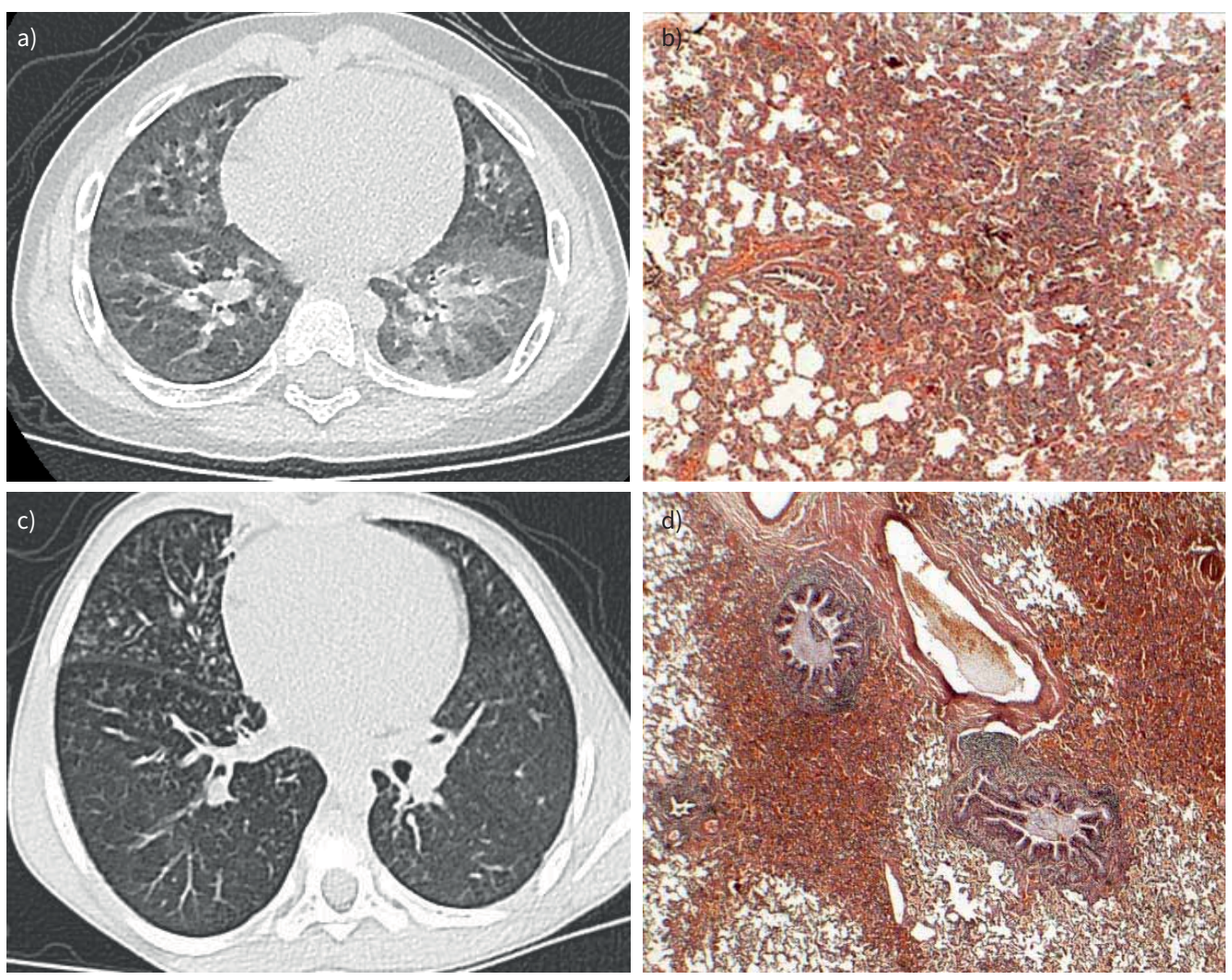

FIGURE 7 a, b) Subject 13: Male patient referred to our hospital by the age of 8 years with history of worsening dyspnoea and bouts of cyanosis. He had a history of recurrent attacks of dyspnoea and dry cough since birth, in addition to global developmental delay of unidentified aetiology. An initial diagnosis of bronchial asthma was made in primary care, yet there was no significant improvement on conventional asthma management. On presentation to us, he was tachypnoeic and hypoxic loxygen saturation $89 \%$ in room air, at rest). Examination revealed marked failure to thrive, in addition to fine crepitaions and wheeze by auscultation. a) Axial computed tomography (CT) shows multiple scattered patchy areas of ground-glass opacities alternating with few areas of air trapping, giving a mosaic pattern; b) histopathology shows chronic bronchiolitis (haematoxylin and eosin $\times 40$ ). c, d) Subject 14. c) Axial CT shows bilateral scattered tiny pulmonary ground-glass nodules 2-3 $\mathrm{mm}$ on average showing centrilobular and peribronchial distribution; d) histopathology shows chronic bronchiolitis with airway lumens focally containing mucin (haematoxylin and eosin $\times 20$ ).

Lung histopathology confirmed the diagnosis of HP in all three cases, and they improved dramatically on elimination of bird exposure and steroid therapy.

Three patients presented with chILD and were diagnosed with CGD after the diagnostic evaluation. They presented with solely pulmonary manifestations of the disease, with less-evident systemic symptoms. All three had notably severe clinical presentation with hypoxaemia at rest, and two had severe pulmonary hypertension. In addition, they had recurrent pneumonias, associated with persistent elevation in erythrocyte sedimentation rate. ChILD seen in CGD patients could be a sequel to recurrent life-threatening infections, or a result of sterile inflammation due to increased expression of pro-inflammatory molecules in genes encoding polymorphonuclear cells [23, 24]. chILD is considered to be a rare pulmonary complication among CGD patients, with few published case reports worldwide. The diagnosis of CGD in our study was made by DHR testing. However, future molecular and genetic studies are needed to identify the causal mutations. The major change in treatment for cases diagnosed with chILD related to CGD was the addition of prophylactic antibiotics (the most commonly used was trimethoprim-sulfamethoxazole) and antifungals (itraconazole/voriconazole). This is in addition to prompt management of any infection with aggressive antimicrobials. Immune-related complications are managed with corticosteroids and immunomodulatory therapies. Corticosteroids were sufficient in two cases, and mycophenolate mofetil was added in one subject due to poor response to steroids.

Additionally, we report a female subject with confirmed histological diagnosis of granulomatous lymphocytic interstitial lung disease (GLILD), which was not related to common variable immune deficiency (CVID). GLILD has been reported frequently in the literature as a complication of CVID in adults. However, in children many cases were not linked to CVID. Recent data suggest that many children 

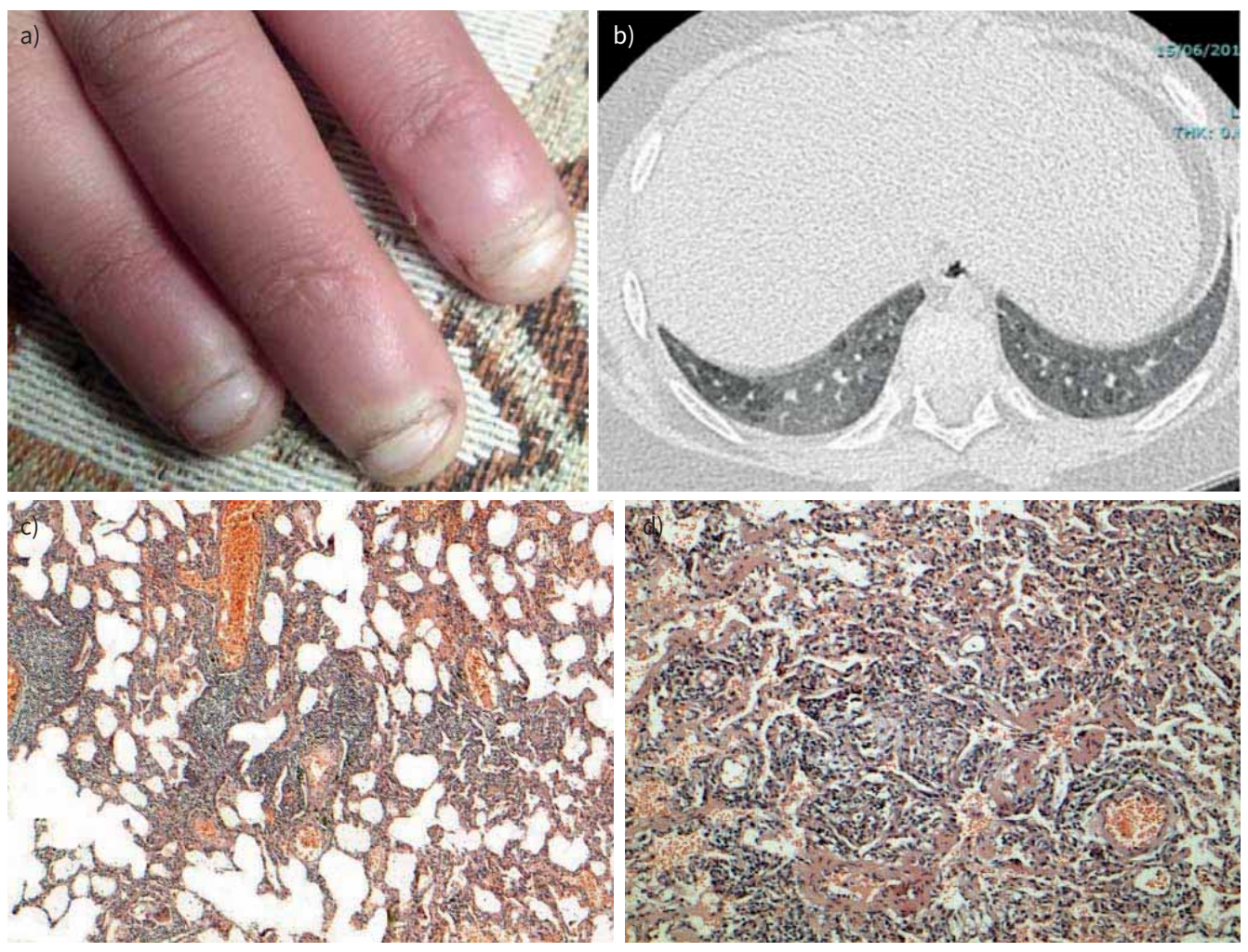

FIGURE 8 Subject 16: 9-year-old female, referred for further assessment of her poorly controlled asthma. On presentation she had dyspnoea and hypoxaemia at rest with widespread fine crepitations and wheeze by auscultation and a) notably puffy fingers. Initial lab investigations were nonconclusive. b) Axial computed tomography shows bilateral mainly lower lobar extensive ground-glassing associated with few areas of air trapping, more evidently affecting the right lower lobe, giving a mosiac pattern. c) Histopathology shows bronchocentric interstitial pneumonia: interstitial chronic inflammation, more marked around bronchovascular bundles (low power); d) an occasional focus of organising pneumonia is noted (haematoxylin and eosin c) $\times 40$ d) $\times 100$ ). In addition, she developed progressive digital tip ulcers, sclerodactyly, in addition to induration proximal to the metacarpophalangeal joint (this appeared later). Overall features confirm the diagnosis of systemic sclerosis.

with GLILD do not fit the CVID criteria and they have a more severe disease in comparison to adults. Additionally, they require intensive treatment with corticosteroids or haemopoietic stem cell transplantation [25]. Our patient showed an initial good response to steroid therapy; however, immunomodulatory therapy was added due to frequent exacerbations.

Among the study group, three subjects had a predominant histological pattern of small airways disease, with a minor component of interstitial pneumonia. Small airways disease has been recently classified as one of the causes of chILD, and it is frequently found to be the sole abnormality in lung biopsy specimens of some chILD patients [9]. Small airways disease can be associated with many diseases, but in many cases the exact cause is not clearly identified. Among our study group the cause of small airways disease couldn't be precisely identified.

We report a female subject who was diagnosed with chILD related to systemic sclerosis. Pulmonary involvement in paediatric systemic sclerosis is common, and it ranges from $30 \%$ to $70 \%$ [26]. Our case had typical radiological features of systemic sclerosis, as her imaging showed ground-glass opacities in a bibasilar distribution. Interestingly, her lung histopathology revealed a rare histological bronchiolocentric pattern of interstitial pneumonia (BPIP). BPIP involves bronchiolocentric fibroinflammatory changes, it is reported to be the result of centrilobular injury due to toxic inhalation or from systemic disease manifesting with airway inflammation, such as collagen diseases [27].

The role of genetic factors in the development of chILD is evident [5]. We observed five cases with history of ILD in closely related family members. One subject diagnosed with CGD after the evaluation had a sibling die of a similar condition. Two cases were siblings, from first-degree consanguineous parents and who had a history of grandparent death in the fourth decade with undiagnosed ILD. The parents agreed a diagnostic lung biopsy for the older sibling only, and it revealed idiopathic BPIP. The exact cause of this 
histopathological pattern could not be specifically identified. BPIP has not been described in a familial setting, but this may reflect some form of genetic predisposition to an exogenous insult. After all, BPIP is still considered a rare subtype of interstitial pneumonia that requires further correlation with clinical studies [28]. The last two cases were diagnosed as interstitial pneumonia by histopathology as fibrotic nonspecific interstitial pneumonia (NSIP) with honeycomb fibrosis, and idiopathic NSIP.

Recently, genetic studies have been recognised as a valuable noninvasive tool in chILD diagnosis. Genetic diagnosis is helpful in estimating recurrence risk and can help avoid lung biopsy [6]. We reported cases with an overall picture suggestive of surfactant protein dysfunction, yet the specific genetic mutation could not be identified due to lack of genetic testing and histopathology electron microscopy studies, which was the major limitation of our study. Future international collaborations will be sought regarding appropriate genetic testing for selected cases.

Among our study group, the diagnostic evaluation changed treatment plans in 13 subjects. The overall diagnostic evaluation helped structure a plan for management and guide prognostic discussions with the parents and changed our perspective in management of subsequent exacerbations.

\section{Conclusion}

We believe this study will help raise awareness of the burden of chILD in Egypt. Furthermore, it highlights the need for the introduction of genetic testing as well as the establishment of a national chILD network, and collaboration with international groups to improve healthcare for Egyptian children with chILD. Lung histopathology provided the most useful diagnostic information which contributed directly to changes in treatment plans, in the absence of genetic testing.

Conflict of interest: S.G. Abdelhady has nothing to disclose. E.M. Fouda has nothing to disclose. M.A. Shaheen has nothing to disclose. F.A. Ghazal has nothing to disclose. A.M. Mostafa has nothing to disclose. A.M. Osman has nothing to disclose. A.G. Nicholson reports personal fees for consultancy on idiopathic pulmonary fibrosis (IPF) trials and education from Boehringer Ingelheim, and personal fees for consultancy on IPF trials from Medical Quantitative Image Analysis and Galapagos, outside the submitted work. H.M. Hamza has nothing to disclose.

\section{References}

1 Griese M. Chronic interstitial lung disease in children. Eur Respir Rev 2018; 27: 170100.

2 Bush A, Cunningham S, de Blic J, et al. European protocols for the diagnosis and initial treatment of interstitial lung disease in children. Thorax 2015; 70: 1078-1084.

3 Kitazawa H, Kure S. Interstitial lung disease in childhood: clinical and genetic aspects. Clin Med Insights Circ Respir Pulm Med 2015; 9: 57-68.

4 Semple TR, Ashworth MT, Owens CM. Interstitial lung disease in children made easier ... well, almost. Radiographics 2017; 37: 1679-1703.

5 Vece TJ, Young LR. Update on diffuse lung disease in children. Chest 2016; 149: 836-845.

6 Kurland G, Deterding RR, Hagood JS, et al. An official American Thoracic Society clinical practice guideline: classification, evaluation, and management of childhood interstitial lung disease in infancy. Am J Respir Crit Care Med 2013; 188: 376-394.

7 Fan LL, Deterding RR, Langston C. Pediatric interstitial lung disease revisited. Pediatr Pulmonol 2004; 38 369-378.

8 Deutsch GH, Young LR, Deterding RR, et al. Diffuse lung disease in young children: application of a nove classification scheme. Am J Respir Crit Care Med 2007; 176: 1120-1128.

9 Rice A, Tran-Dang M, Bush A, et al. Diffuse lung disease in infancy and childhood: expanding the chILD classification. Histopathology 2013; 63: 743-755.

10 Nathan N, Berdah L, Borensztajn K, et al. Chronic interstitial lung diseases in children: diagnosis approaches Expert Rev Respir Med 2018; 12: 1051-1060.

11 Clement A, Nathan N, Epaud R, et al. Interstitial lung diseases in children. Orphanet J Rare Dis 2010; 5: 22.

12 Cunningham S, Graham C, MacLean M, et al. One-year outcomes in a multicentre cohort study of incident rare diffuse parenchymal lung disease in children (ChILD). Thorax 2020; 172-175.

13 Saddi V, Beggs S, Bennetts B, et al. Childhood interstitial lung diseases in immunocompetent children in Australia and New Zealand: a decade's experience. Orphanet J Rare Dis 2017; 12: 133.

$14 \mathrm{Xu} \mathrm{D}$, Chen $\mathrm{Z}$, Chen $\mathrm{H}$, et al. Application of clinico-radiologic-pathologic diagnosis of diffuse parenchymal lung diseases in children in China. PLoS One 2015; 10: e0116930.

15 Parris D, van Niekerk A, Jeevarathnum AC, et al. An approach to pulmonary haemorrhage in children. S Afr Respir J 2017; 23: 63-70.

16 Greenhalgh R, Yardley I, Child F, et al. Lung biopsy for chronic pulmonary disease in children. J Pediatr Surg 2014; 49: 1075-1077.

17 Hafezi N, Heimberger M, Lewellen K, et al. Lung biopsy in children's interstitial and diffuse lung disease: does it alter management? Pediatr Pulmonol 2020; 55: 1050-1060.

18 O'Reilly R, Kilner D, Ashworth M, et al. Diffuse lung disease in infants less than 1 year of age: histopathological diagnoses and clinical outcome. Pediatr Pulmonol 2015; 50: 1000-1008.

19 Griese M, Haug M, Hartl D, et al. Hypersensitivity pneumonitis: lessons for diagnosis and treatment of a rare entity in children. Orphanet J Rare Dis 2013; 8: 121. 
Soumagne T, Dalphin M, Dalphin J. Pneumopathie d'hypersensibilité de l'enfant. [Hypersensitivity pneumonitis in children]. Rev Mal Respir 2019; 36: 495-507.

21 Millerick-May M, Mulks M, Gerlach J, et al. Hypersensitivity pneumonitis and antigen identification - an alternate approach. Respir Med 2016; 112: 97-105.

22 Ratjen F, Costabel U, Griese M, et al. Bronchoalveolar lavage fluid findings in children with hypersensitivity pneumonitis. Eur Respir J 2003; 21:144-148.

23 Thomsen IP, Smith MA, Holland SM, et al. A comprehensive approach to the management of children and adults with chronic granulomatous disease. J Allergy Clin Immunol Pract 2016; 4: 1082-1088.

24 Mortaz E, Azempour E, Mansouri D, et al. Common infections and target organs associated with chronic granulomatous disease in Iran. Int Arch Allergy Immunol 2019; 179: 62-73.

25 Nademi Z, Davies G, Devlin L, et al. 144 Granulomatous lymphocytic interstitial lung disease (GLILD) in children. Arch Dis Child 2018; 103: A58.

26 Torok K. Pediatric scleroderma: systemic or localized forms. Pediatr Clin North Am 2012; 59: 381-405.

27 Tanaka T, Ishida K. Update on rare idiopathic interstitial pneumonias and rare histologic patterns. Arch Pathol Lab Med 2018; 142: 1069-1079.

28 Travis WD, Costabel U, Hansell DM, et al. An official American Thoracic Society/European Respiratory Society statement: update of the international multidisciplinary classification of the idiopathic interstitial pneumonias. $A m$ J Respir Crit Care Med 2013; 188: 733-748. 